\title{
Omalizumab in an allergology clinic: real life experience and future developments
}

\author{
Maciej Kupczyk, Piotr Kuna \\ Department of Internal Medicine, Asthma and Allergy, Medical University of Lodz, Poland \\ Head of Department: Piotr Kuna MD, PhD
}

Postep Derm Alergol 2014; XXXI, 1: 32-35

DOI: $10.5114 /$ pdia.2014.40657

\begin{abstract}
Omalizumab is a recombinant humanized monoclonal antibody that reduces levels of circulating IgE and expression of IgE high affinity receptor ( $\mathrm{FCERI}$ ) on mast cells and basophils. Its role in the therapy of allergic asthma and urticaria is well established. According to GINA guidelines, omalizumab should be considered as an important alternative to systemic corticosteroids in uncontrolled asthma. Several ongoing trials will evaluate omalizumab efficacy in the treatment of other allergic diseases and conditions. Further studies are needed to answer several practical questions on the optimal duration of treatment and possible biomarkers to predefine a cohort of responders to this therapy.
\end{abstract}

Key words: omalizumab, immunoglobulin E, allergic asthma, urticaria.

Omalizumab is a recombinant humanized monoclonal antibody that selectively binds to the $C_{\varepsilon} 3$ domain of free immunoglobulin E (IgE), forming complexes with circulating IgE and reducing its levels. By preventing IgE interaction with the high-affinity IgE receptor (FCERI) on mast cells and basophils, release of soluble pro-inflammatory mediators is decreased. Moreover, a reduction in free IgE leads to significant down-regulation of the FCERI expression on inflammatory cells and reduction in cells' influx (including eosinophils) to the site of inflammation. Subsequently, allergic inflammation is modulated and symptoms of allergic diseases are decreased. Omalizum$a b$ is administered subcutaneously in doses dependent on the baseline total IgE level and body mass. In Europe, omalizumab is currently approved for the treatment of children, adolescents and adults (aged 6 years and above) with severe allergic asthma in whom symptoms are not well controlled despite high doses of inhaled corticosteroid (ICS) and long acting $\beta_{2}$ agonist. In the US, omalizumab is recommended in the treatment of adults and adolescents (aged 12 years and above) with moderate to severe persistent asthma whose symptoms are poorly controlled with ICS. According to GINA guidelines [1], omalizumab should be considered at step 5 of therapy as an important alternative to systemic corticosteroids. The burden of frequent adverse events induced by systemic corticosteroids (SC), including but not limited to osteoporosis, glaucoma, cataract, diabetes, obesity, skin thinning, easy bruising, arterial hypertension and muscle weakness, significantly limits its use in high doses in long-term treatment. It should be underlined here that GINA guidelines clearly recommend omalizumab as an alternative to systemic corticosteroids, not as a next step of therapy when SCs are ineffective. This is in contrast to common clinical practice as omalizumab is often added to therapy in the most severe patients with frequent exacerbations and on high doses of CS/ICS/SC. The proportion of SC and omalizumab as a first choice step 5 treatment in asthma will change as the access to this drug is increasing.

Several long-term, randomized, controlled trials and pragmatic, real-life observations confirmed the benefits of omalizumab in moderate to severe allergic asthma. A systematic review published in 2011 [2] included 8 trials and in total 3429 subjects with asthma. Patients taking omalizumab were more likely to be able to step down in the dosing of inhaled corticosteroids in comparison to those on placebo (relative risk $(R R)=1.80, p<0.00001$ ). Omalizumab significantly decreased the risk of asthma exacerbations $(R R=0.57)$. This is in line with the previous report of the Cochrane review published in 2006 [3] of 14 trials in 3143 children and adults with asthma

Address for correspondence: Maciej Kupczyk MD, PhD, Department of Internal Medicine, Asthma and Allergy, Medical University of Lodz, 22 Kopcinskiego St, 90-153 Lodz, Poland, phone: +48 4267766 49, e-mail: maciek.kupczyk@ki.se Received: 7.01.2014, accepted: 9.01.2014. 
where omalizumab was found to reduce the number of asthma exacerbations $(O R=0.52)$ and increase the proportion of patients who were able to reduce the intensity of treatment. Braunstahl et al. [4] evaluated the efficacy of omalizumab in the real-world clinical practice including 943 patients into the open-label observational registry. Omalizumab was found to significantly increase the proportion of patients with no clinically important exacerbations from $6.8 \%$ at baseline to $54.1 \%$ and $67.3 \%$ at month 12 and 24 , respectively of follow up. Symptoms and rescue medication use were reduced by more than $50 \%$ from baseline. Maintenance CS use decreased to $14.2 \%$ at month 24 as compared to $28.6 \%$ at baseline [5]. As mentioned above, omalizumab is currently licensed for the treatment of uncontrolled allergic asthma. However, as reviewed by Babu et al. [6], there are 109 ongoing trials in 46 different diseases and conditions other than asthma. Among those, some reports showed benefits of omalizumab in seasonal and allergic rhinitis, atopic dermatitis, urticaria, idiopathic anaphylaxis, peanut allergy, latex allergy, mastocytosis, eosinophilic gastroenteritis, bronchopulmonary aspergillosis and nasal polyposis. What is of special interest, clinical trials analyzing efficacy of omalizumab in non-allergic asthma are underway. Preliminary results suggest that omalizumab will become a promising treatment option in this cohort of patients as well.

In the latest systematic review [2] presented above, the frequency of adverse events was similar in the omalizumab (3.8\%) and placebo (5.3\%) groups with the only difference in injection site reactions (limited erythema, wheal), which were more frequent in the omalizumab patients (19.9\% vs. 13.2\%). Authors of this meta-analysis concluded that there were no indications of an increased risk of hypersensitivity reactions, cardiovascular effects or malignant neoplasms. The incidence of anaphylaxis after omalizumab injection varies from $0.14 \%$ to $0.2 \%$ [7]. Majority of events were reported after the first 3 doses of omalizumab and developed within $2 \mathrm{~h}$ following the injection. Thus, it is highly recommended that the patient should be under follow up at the site well equipped and ready for the treatment of anaphylaxis for $2 \mathrm{~h}$ following the first 3 injections and for 30 min for the subsequent injections. Some ambiguity regarding an increased risk of malignancies in omalizumab treated paints was raised. In the latest pooled analysis [8] of data from 67 clinical trials including 7789 patients receiving omalizumab, 25 malignancies were identified, 14 in the omalizumab and 11 in the placebo treated group. Malignancies were of varying histological type and occurred in a number of different organs. Thus, authors of the analysis concluded that the causal relationship between omalizumab treatment and malignancy is unlikely. Studies [9] addressing concerns of the increased risk of cardiovascular and cerebrovascular events in omalizumab treated patients are ongoing and final reports should be available soon.
Taking into account the real-life experience with omalizumab, several practical questions arise. As reported by Braunstahl et al. [5], the efficacy of omalizumab treatment is good in majority of patients with uncontrolled asthma. Among 915 patients receiving omalizumab included into the study, $64.2 \%$ were responders (defined as excellent or good response), however $30.7 \%$ were non-responders (moderate, poor response or worsening). None of analyzed immunological or clinical factors consistently correlated with therapeutic response to omalizumab in asthma, thus there are currently no biomarkers or clinical characteristics that can be used as a predictor of good response to the treatment. Nothing more than detailed assessment of efficacy at week 16 (as currently indicated in several position papers) and later during the follow up can be recommended. This assessment should include measures of asthma control, quality of life, medication use (including inhaled, oral corticosteroids and rescue medication use), and number of exacerbations.

Another practical issue is the duration of treatment. This should not be surprising that taking into account costs of omalizumab therapy, several studies assessing cost-effectiveness of omalizumab are published [10,11] and the national health service authorities pursue ideas of stopping the therapy after some period of time. Careful re-evaluation of symptoms of the underlying disease after the break in the treatment may lead to re-introduction of omalizumab, which is the case in the majority of patients in our clinical practice. It needs to be clearly said that there are no published trials evaluating the duration of treatment with omalizumab. Reports published to date include data from the randomized trials of 24 to 60 weeks' duration on average. It is a well-known fact that after cessation of omalizumab therapy symptoms slowly arise and the controller medication use increases in asthmatic subjects. In the current issue of the "Postępy Dermatologii i Alergologii", Kuprys et al. [12] reports on the loss of asthma control after cessation of omalizumab treatment. Data from studies in urticaria are even more striking than the observations of asthma cohorts. Kaplan et al. [13] found that symptoms of urticaria flared up to levels seen in the placebo group within 16 weeks of follow up after the end of highly effective treatment with omalizumab. Similarly, in the study of Maurer et al. [14] symptoms of urticaria reached levels seen in the placebo group within 4 to 10 weeks after the cessation of omalizumab treatment. What was interesting, the time of symptoms recurrence was reversibly dependent on the dose of omalizumab and thus its efficacy during the treatment phase.

One can hypothesize that the efficacy of therapy with omalizumab and the duration of treatment may be dependent on the underlying condition (asthma, urticaria, atopic dermatitis or other), severity of the disease (severe vs. mild) and some immunological factors (baseline total IgE levels or other currently unknown biomarkers). 
Appropriate diagnosis and treatment of co-morbidities in severe, uncontrolled asthma (including chronic rhinosinusitis, reflux disease, sleep apnea, obesity, infections) and ability to decrease exposure to triggering factors (including but not restricted to allergens, non-steroidal anti-inflammatory drugs, and pollution) may have a significant impact on the level of disease control and thus may modulate the time of omalizumab treatment. Natural history of the disease needs to be taken into consideration. Again, one can suggest that in patients with severe asthma, which is usually regarded as a stable phenotype of the disease, the treatment duration may need to be longer than in chronic urticaria where the symptoms significantly improve in majority of patients within 1 to 2 years of follow up. Nopp et al. [15] evaluated the level of asthma control after cessation of treatment in a small cohort of 18 patients suffering from severe cat allergen-induced asthma. Three years after a 6-year period of effective omalizumab treatment, majority of patients (12 among 18) reported improved or unchanged asthma symptoms, 16 of 18 had no increase in night attacks and 14 of the cohort had little or no increase in the asthma medication use. In another paper, Lowe et al. [16] presented a pharmacokinetic-pharmacodynamic model based on data compiled from 1 epidemiological study and 6 randomized trials in patients with asthma with a goal to evaluate changes in the IgE production over time in subjects on omalizumab therapy. It has been found that IgE production decreases on average by $54 \%$ per year and it has been estimated that it would take 3-5 years, for the responders, to reach a new steady state. Total IgE initially increases, due to formation of omalizumab-lgE complexes, reaching its peak at weeks 12-16, then slowly decreases. Monitoring of total IgE in a longterm treatment may be a promising marker of IgE production. On treatment withdrawal, IgE production should begin to increase, but more slowly, taking approximately 15 years to return to baseline levels. Authors concluded that it is possible that treatment with omalizumab does not need to be indefinite and maintained for long periods of time. Molimard et al. [17] presented data from a cohort of 61 asthma patients with mean duration of omalizumab treatment summing up to 22.7 months. After discontinuation of omalizumab, the percentage of patients losing control was $69.2 \%, 59.1 \%$ and $45.7 \%$ after $<1$ year, $1-2$ years and $>2$ years of treatment, respectively. Reintroduction of omalizumab was successful in majority of patients (14 out of 20). The results of this report suggest that the number of patients with loss of asthma control decreases with time of effective omalizumab treatment. Taking into consideration available data, our clinical experience with omalizumab treatment and natural history of allergic diseases, it may be hypothesized that the optimal timing for re-evaluation of indications to omalizumab therapy may be 5 to 6 years in severe allergic asthma and 1 to 2 years in urticaria. However, it must be clear that there are definitely too few studies to draw final conclusions and moreover, the proposed timing may be highly variable in particular patients as several factors influence both the response to omalizumab therapy and level of the disease control.

As mentioned above, the usefulness of omalizumab in the treatment of several other conditions than asthma, including chronic or spontaneous urticaria, is being evaluated. First reports on efficacy of omalizumab in patients suffering from urticaria come from case reports, small trials or case series [18]. In this issue of the "Postępy Dermatologii i Alergologii", Kuprys et al. [19] present yet another case report on effectiveness of omalizumab in a patient with severe asthma, urticaria and angioedema. In a randomized, placebo-controlled, dose-ranging study of a single dose of omalizumab, Saini et al. [20] found that a fixed dose of $300 \mathrm{mg}$ or $600 \mathrm{mg}$ of omalizumab provides rapid and effective treatment of chronic idiopathic urticaria in patients who are symptomatic despite treatment with $\mathrm{H}_{1}$-antihistamines. Kaplan et al. [13] aimed to evaluate safety and efficacy of omalizumab in patients with chronic urticaria symptomatic despite treatment with $\mathrm{H}_{1}$-antihistamines at up to 4 times the approved dose plus $\mathrm{H}_{2}$-antihistamines, leukotriene receptor antagonists, or both. More than 250 patients received 6 subcutaneous injections of $300 \mathrm{mg}$ of omalizumab at 4-week intervals. At week 12, the mean change from baseline in weekly itch severity score was -8.6 (95\% Cl: -9.3 to -7.8$)$ in the omalizumab group compared with $-4.0(95 \% \mathrm{Cl}$ : -5.3 to -2.7$)$ in the placebo group $(p<0.001)$. The overall incidence and severity of adverse events were similar in omalizumab and placebo recipients. In the study of Maurer et al. [14], 323 patients with chronic urticaria symptomatic despite recommended treatment were randomly assigned to receive 3 injections of $75 \mathrm{mg}, 150 \mathrm{mg}$, or 300 mg of omalizumab or placebo. Both itch severity score and number of hives decreased significantly in the dose-dependent manner in the omalizumab treated patients. In conclusion, omalizumab has been found to be highly effective and well-tolerated therapy in patients suffering from uncontrolled chronic urticaria. Taking the results of randomized controlled trials published to date into consideration, in the latest revision of the international guidelines on the diagnosis and therapy of chronic urticaria [21], omalizumab has been proposed as the first-line treatment of refractory patients. There are no doubts that soon omalizumab will be licensed in several European countries in this condition as well.

Omalizumab represents a breakthrough in the pharmacotherapy of uncontrolled allergic asthma and chronic urticaria. This humanized monoclonal antibody is safe and well tolerated. Several ongoing trials will evaluate its efficacy in other allergic diseases and conditions. There are no doubts that the role of omalizumab is well established. However, further studies are needed to answer several practical questions on the optimal duration of 
treatment and possible biomarkers to predefine a cohort of responders to this therapy.

\section{References}

1. Global Initiative for Asthma. Global strategy for asthma management and prevention updated 2012. Available online at http://www.ginasthma.com

2. Rodrigo GJ, Neffen H, Castro-Rodriguez JA. Efficacy and safety of subcutaneous omalizumab vs placebo as add-on therapy to corticosteroids for children and adults with asthma: a systematic review. Chest 2011; 139: 28-35.

3. Walker S, Monteil M, Phelan K, et al. Anti-IgE for chronic asthma in adults and children. Cochrane Database Syst Rev 2006; 2: CD003559.

4. Braunstahl GJ, Chen CW, Maykut R, et al. The eXpeRience registry: the real-world effectiveness of omalizumab in allergic asthma. Resp Med 3013; 107: 1141-51.

5. Braunstahl GJ, Chlumsky J, Peachey G, Chen CW. Reduction in oral corticosteroid use in patients receiving omalizumab for allergic asthma in the real-world setting. Allergy Asthma Clin Immunol 2013; 9: 47.

6. Babu KS, Polosa R, Morjaria JB. Anti-lgE - emergin opportunities for omalizumab. Expert Opin Biol Ther 2013; 13: 765-77.

7. Thomson NC, Chaudhuri R. Omalizumab: clinical use for the management of asthma. Clin Med Insights Circ Respir Pulm Med 2012; 6: 27-40.

8. Busse W, Buhl R, Fernandez Vidaurre C, et al. Omalizumab and the risk of malignancy: results from a pooled analysis. J Allergy Clin Immunol 2012; 129: 983-9.

9. Long AA, Fish JE, Rahamaoui A, et al. Baseline characteristics of patients enrolled in EXCELS: a cohort study. Ann Allergy Asthma Immunol 2009; 103: 312-9.

10. Campbell JD, Spackman DE, Sullivan SD. The costs and consequences of omalizumab in uncontrolled asthma from a USA payer perspective. Allergy 2010; 65: 1141-8.

11. Brown R, Turk F, Dale P, Bousquet J. Cost-effectiveness of omalizumab in patients with severe persistent allergic asthma. Allergy 2007; 62: 149-53.

12. Kuprys-Lipinska I, Kuna P. Loss of asthma control after cessation of omalizumab treatment: real life data. Postep Derm Alergol 2014; 31: 1-5.

13. Kaplan A, Ledford D, Ashby M, et al. Omalizumab in patients with symptomatic chronic idiopathic/spontaneous urticaria despite standard combination therapy. J Allergy Clin Immunol 2013; 132: 101-9.

14. Maurer M, Rosen K, Hsieh HJ, et al. Omalizumab in the treatment of chronic idiopathic or spontaneous urticaria. N Engl J Med 2013; 368: 924-35.

15. Nopp A, Johansson SG, Adedoyin J, et al. After 6 years with Xolair: a 3-year withdrawal follow-up. Allergy 2010; 65: 56-60.

16. Lowe PJ, Renard D. Omalizumab decreases IgE production in patients with allergic (IgE-mediated) asthma: PKPD analysis of a biomarker, total IgE. Br J Clin Pharmacol 2011; 72: 306-20.

17. Molimard M, Le Gros V, Bourdeix I, et al. Observational study in severe asthmatic patients after discontinuation of omalizumab for good asthma control. European Respiratory Society 2013 Congress. Abstract No. 4693. Abstract book 856s.

18. Metz M, Maurer M. Omalizumab in chronic urticaria. Curr Opin Allergy Clin Immunol 2012; 12: 406-11.
19. Kuprys-Lipinska I, Korczynska P, Tworek D, Kuna P. Effectiveness of omalizumab in a patient with a life-threatening episode of bronchospasm and larynx angioedema after exposure to house dust. Postep Derm Alergol 2014; 31: 39-44.

20. Saini S, Rosen KE, Hsieh HJ, et al. A randomized, placebo-controlled, dose-ranging study of single-dose omalizumab in patients with $\mathrm{H1}$-antihistamine-refractory chronic idiopathic urticaria. J Allergy Clin Immunol 2011; 128: 567-73.

21. Maurer M, Magerl M, Metz M, Zuberbier T. Revisions to the international guidelines on the diagnosis and therapy of chronic urticaria. J Germ Soc Dermatol 2013 in press. DOI: 10.1111/ddg.12194. 